Article

\title{
Role of DTAB and SDS in Bubble-Particle Attachment: AFM Force Measurement, Attachment Behaviour Visualization, and Contact Angle Study
}

\author{
Yaowen Xing ${ }^{1,2, *}$, Mengdi Xu ${ }^{1,3}$, Ming Li $^{3}$, Wei Jin ${ }^{3}$, Yijun Cao ${ }^{2}$ and Xiahui Gui ${ }^{1,2, *}$ \\ 1 Key Laboratory of Coal Processing and Efficient Utilization of Ministry of Education, China University \\ of Mining and Technology, Xuzhou 221116, China; cumtxmd@outlook.com \\ 2 Chinese National Engineering Research Center of Coal Preparation and Purification, China University \\ of Mining and Technology, Xuzhou 221116, China; yijuncao@126.com \\ 3 School of Chemical Engineering and Technology, China University of Mining and Technology, \\ Xuzhou 221116, China; 15062114600@163.com (M.L.); zgkydxjin@163.com (W.J.) \\ * Correspondence: cumtxyw@cumt.edu.cn (Y.X.); guixiahui1985@163.com (X.G.); \\ Tel.: +86-0516-8359-1116 (Y.X. \& X.G.)
}

Received: 19 July 2018; Accepted: 7 August 2018; Published: 13 August 2018

\begin{abstract}
Atomic force microscopy (AFM) and contact angle measurements were used to study the role of dodecyltrimethylammonium bromide (DTAB) and sodium dodecyl sulphate (SDS) in bubble-particle attachment. The results show that the forces between bubbles and the hydrophilic glass particle were always repulsive in the absence of DTAB and SDS. An attractive hydrophobic force was induced when the particles became hydrophobic, and the force was proportional to the water contact-angle. In the presence of DTAB and SDS, the cationic head group of DTAB adsorbed onto the negative hydrophilic glass surface as a monolayer and thus induced a hydrophobic force. However, at a high DTAB concentration, the DTAB molecules began to adsorb as a bilayer, reverting back to a hydrophilic surface. The hydrophobic force disappeared and the water film between the bubble and particle was stabilised under the repulsive double-layer force. The anionic SDS molecules could not adsorb onto the hydrophilic glass surface. The repulsive force always dominated the bubble-particle interaction. In the case of hydrophobic glass, the hydrophobic force decreased, and even disappeared, with the addition of DTAB and SDS. All the findings from the AFM force curves were consistent with the attachment behaviour and contact angle results.
\end{abstract}

Keywords: flotation; bubble-particle attachment; DTAB; SDS; AFM; contact angle

\section{Introduction}

Froth flotation is one of the most commonly used separation methods for fine particles and is based on the difference in wetting properties [1-4]. It is always used for concentrating mineral particles below $0.5 \mathrm{~mm}$ in the mining industry. Before flotation, crushing and grinding are necessary steps to achieve the liberation of the valuable components from the gangue [5]. Then, these fine particles are mixed with water and conditioned with appropriate reagents. Air is introduced, and selective attachment between the targeted particles and bubbles is finally achieved. Bubble-particle attachment is the critical step for successful flotation [1,6,7]. In normal cases, only hydrophobic particles can be captured by the rising bubbles in a flotation cell $[8,9]$.

Most minerals are not naturally hydrophobic. A surfactant, also called a collector, is always added to improve the surface hydrophobicity in flotation processes. Consequently, bubble-particle attachment becomes possible [10]. Studies have shown that the fundamental role of the collector is to induce an attractive hydrophobic force and thus overcome the repulsive Derjaguin-Landau-Verwey-Overbeek 
(DLVO) forces, including the van der Waals force and electrostatic force [1,2,10-12]. Using atomic force microscopy (AFM), Butt [13] was the first to measure the force between a hydrophilic/hydrophobic glass sphere and an air bubble. It was found that hydrophilic particles experienced a repulsive force, while hydrophobic particles experienced an attractive force. Nguyen et al. [14,15] used AFM to measure the force between a hydrophilic particle and a bubble, with different approach speeds. The repulsive force was found to increase monotonically with increasing approach speed. However, these studies only focused on systems without surfactants. In the presence of surfactants, due to the strong deformation of the bubble interface and the adsorption of surfactants, a strong attractive force may be difficult to measure due to the limitation of the AFM piezo displacement and split photodiode. Preuss and Butt [16] established a home-made setup based on the principle of commercial AFM to study the influence of surfactants (dodecyltrimethylammonium bromide (DTAB) and sodium dodecyl sulphate (SDS)) on the bubble-particle interaction force. The diameter of the microsphere was $5 \mu \mathrm{m}$. The force results could be interpreted according to the DLVO and extended DLVO theories. However, due to the complexity of this system, the role of different surfactants in bubble-particle attachment is still not well-understood.

In this work, we further measured the interaction force between an air bubble and a larger micrometre sphere ( $40 \mu \mathrm{m}$ diameter), compared with that in Preuss and Butts' work [16], in the presence of the two surfactants, DTAB and SDS. The reason for choosing DTAB and SDS is that the properties of both these surfactants are well-studied. Their critical micelle concentrations are 16 and $8 \mathrm{mM}$ for DTAB and SDS, respectively. A commercial NanoWizard ${ }^{\mathrm{TM}}$ III AFM instrument (JPK Instruments) with a large piezo range $(15 \mu \mathrm{m})$ was used. In addition, an in-house-developed attachment behaviour visualisation system and contact angle meter were used to obtain complementary information. The outcomes of this study are expected to provide a basic understanding of the bubble-particle attachment mechanism in surfactant systems.

\section{Materials and Methods}

\subsection{Materials}

The glass microspheres used for preparing the AFM colloidal probe were purchased from Duke Scientific Corporation, Palo Alto, CA, USA. The SEM images of the glass microspheres are shown in Figure 1. The surface of the microsphere was quite smooth, and hence, the surface roughness effect in AFM force measurement could be neglected. DTAB, SDS, and octadecyltrichlorosilane (OTS) were purchased from Sigma-Aldrich, Oakville, ON, Canada. Milli-Q water was used in all the experiments.
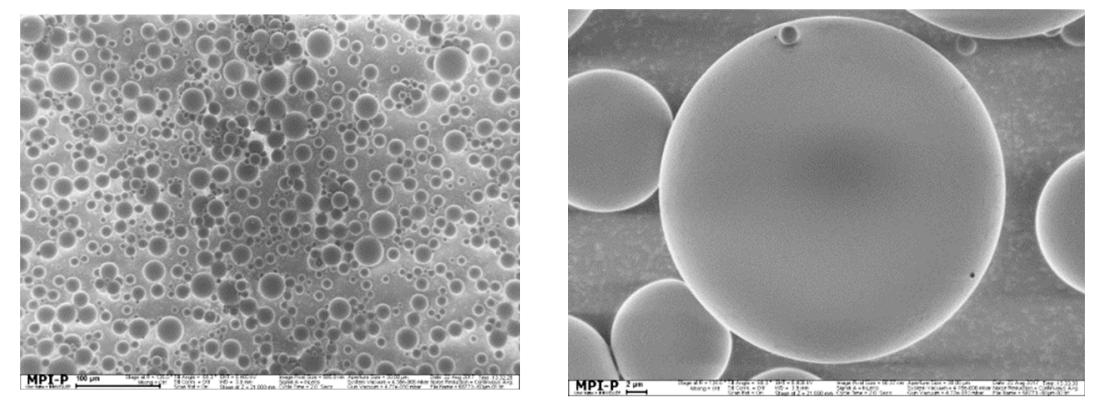

Figure 1. SEM images of the glass microspheres.

\subsection{Methods}

\subsubsection{AFM Force Measurements}

Glass microspheres with about $40 \mu \mathrm{m}$ diameter were select to prepare the colloidal probe. Force measurements between the bubbles and glass particles in the presence of DTAB and SDS were carried 
out in a glass dish using a NanoWizard ${ }^{\mathrm{TM}}$ III AFM instrument (JPK Instruments AG, Berlin, Germany). A hydraulic micromanipulator (MMO-203, Narishige Co, LTD, Tokyo, Japan) was used for preparing the glass colloidal probe under an optical microscope (Zeiss Axiotech, Carl Zeiss AG, Oberkochen, Germany). A tip-less cantilever with a $0.65 \mathrm{~N} / \mathrm{m}$ spring constant was mounted at the clamp of the micromanipulator and then attached to a glass sphere with the aid of epoxy resin [17]. An air bubble with a 300-600 $\mu \mathrm{m}$ diameter was injected onto a hydrophobic glass dish after OTS hydrophobisation, using a micro syringe. Before the force experiments, the sensitivity and spring constant of the colloidal probe were calibrated by the Sader method. The colloidal probe was cleaned with Argon plasma for $10 \mathrm{~min}$ to remove any potential organic contamination. After plasma treatment, the glass sphere should be completely hydrophilic. For the hydrophobic colloid probe, we placed the hydrophilic colloid probe under OTS vapour. Different contact angles could be obtained by controlling the treatment time. After the silanisation, chloroform, acetone, ethanol, and Milli-Q water were used to rinse the excess OTS molecules on the particle surface. The probe was finally mounted and positioned at the top of air bubble with the help of an optical microscope. After pre-setting the set-point and approach speed, force curves were obtained by recording the cantilever deflection using an optically sensitive lever technique. All the experiments were conducted at room temperature $\left(20^{\circ} \mathrm{C}\right)$.

The raw AFM data obtained was a force-displacement curve. Therefore, it is necessary to convert the AFM force-displacement curve into a force-distance curve. During this conversion, the critical point is to determine the zero distance [18-21]. In a hard contact system, the zero distance can be obtained from the nominal compliance regime. For bubble-particle interactions, the deformation of the air-water interface under the applied force is a problem. There is no hard contact point in such cases. Earlier studies have shown that the equilibrium position of a particle trapped in the bubble surface could be set as the zero distance $[20,21]$. Here, the bubble was assumed to behave as a Hookean spring with a spring constant $\mathrm{k}_{\mathrm{b}}$ under external force. A linear compliance region also existed when the particle was approached by the bubble surface. Then, the slope of the nominal constant-compliance regime in the force-displacement curve is equal to the spring constant of the bubble-cantilever system. A schematic of the bubble-particle interaction in the AFM system is shown in Figure 2. The initial distance between the bubble and particle is denoted as $\mathrm{D}_{0}$, and $\mathrm{y}_{0}$ is the bubble height. When the bubble approaches the particle $(z)$, the cantilever bends upward (d) due to the interaction force. Based on the principle of force balance, the following Equation can be obtained:

$$
\mathrm{k}_{\mathrm{c}} \mathrm{d}=\mathrm{k}_{\mathrm{b}}\left(\mathrm{y}_{0}-\mathrm{y}\right)
$$

where $\mathrm{k}_{\mathrm{c}}$ is the spring constant of the cantilever.

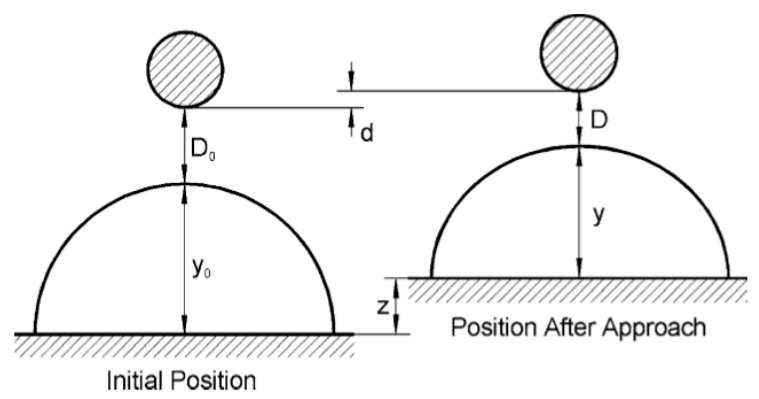

Figure 2. Schematic of bubble-particle interaction in AFM system [15].

From Figure 2, we obtain:

$$
\mathrm{D}_{0}+\mathrm{y}_{0}+\mathrm{d}=\mathrm{z}+\mathrm{y}+\mathrm{D}
$$


Combining Equations (1) and (2) gives:

$$
\mathrm{D}=\mathrm{d}\left(1+\mathrm{k}_{\mathrm{c}} / \mathrm{k}_{\mathrm{b}}\right)-\mathrm{z}+\mathrm{D}_{0}
$$

Equation (3) indicates that the variation of the separation distance is equal to the displacement of the AFM piezo minus the deformations of both the bubble and cantilever. The detailed conversion principle is available in the literature $[15,20,21]$. Each force experiment was repeated at least five times, and the general trend force curve is shown in the following section.

\subsubsection{Attachment Behaviour Visualisation and Contact Angle Measurement}

An in-house-developed high-speed visualisation system was used to monitor the attachment behaviour between the bubble and a glass slide in the presence of DTAB or SDS. The system is similar to a traditional induction timer. The detailed information about the high-speed visualisation system can be found in [17]. The minimum contact time for attachment to be observed was defined as the induction time. During the experiment, the bubble diameter, bubble displacement, initial distance gap, and approach velocity were all held constant. The sessile drop method was applied to measure the water contact-angle on the glass slide.

\section{Results and Discussion}

\subsection{Bubble-Particle Interaction Force in the Absence of DTAB and SDS}

A colloidal probe with $40 \mu \mathrm{m}$ diameter was prepared and placed on the top of a bubble, as shown in Figure 3. The interaction force between the hydrophilic glass microsphere and the bubble, for different collision velocities, is shown in Figure 4. The forces were always repulsive for all the approach velocities. There was no successful attachment between the hydrophilic glass and the bubble. This repulsive force increased with the approach velocity due to the appearance of a hydrodynamic force. When the approach velocity was lower than $1 \mu \mathrm{m} / \mathrm{s}$, the hydrodynamic force could be neglected, and only the surface force dominated. According to the DLVO theory, the stability of a colloid system is controlled by two additive components, i.e., the van der Waals force and electrostatic double-layer force. In the bubble-glass system, both the van der Waals and double-layer forces are repulsive, and do not provide any driving force for film thinning and rupture. This is consistent with the AFM force curves.

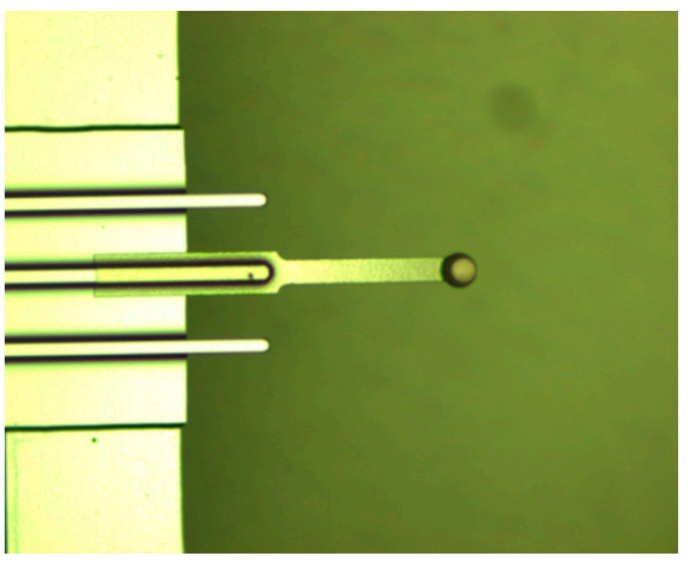

(a)

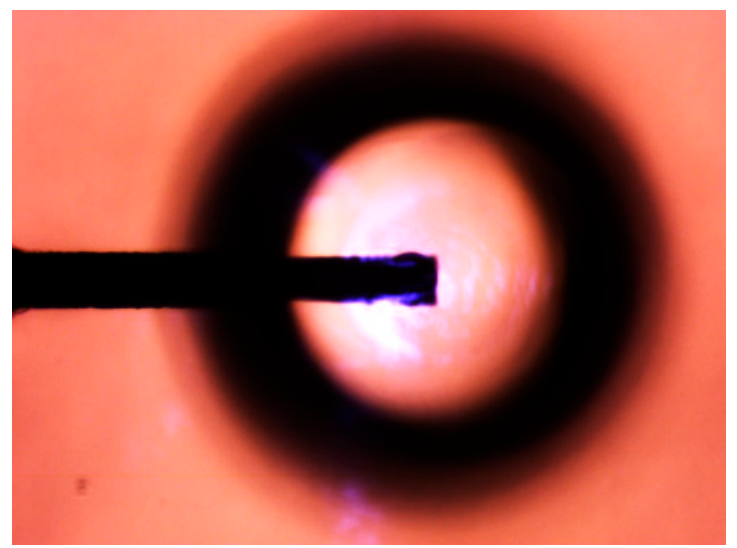

(b)

Figure 3. Colloidal probe of hydrophilic glass microsphere (a); Snapshot of bubble-particle interaction (b).

Colloidal probes with $70^{\circ}$ and $100^{\circ}$ contact angles were prepared by OTS silanisation. In order to estimate the contact angle, a glass slide was hydrophobised simultaneously, with the same procedure as 
that for the glass microsphere, according to the literature [14]. The Washburn method was not applied to measure the particle contact-angle in this study because the amount of glass microspheres in our lab was not sufficient for packing the column and conducting repeated tests. The effect of the contact angle on the interaction force between the glass microsphere and the bubble, with a $1 \mu \mathrm{m} / \mathrm{s}$ approach velocity, is shown in Figure 5. A significant jump-into contact was observed for the hydrophobic particle due to some kind of attractive force. The thin water-film between the hydrophobic particle and the bubble was unstable. The jump-into distance increased with the contact angle. The magnitude of the attractive force was proportional to the water contact-angle. Both the van der Waals and electrical double-layer forces should not be changed after OTS silanisation. Consequently, the hydrophobic force was responsible for the bubble-particle attachment $[1,2]$.

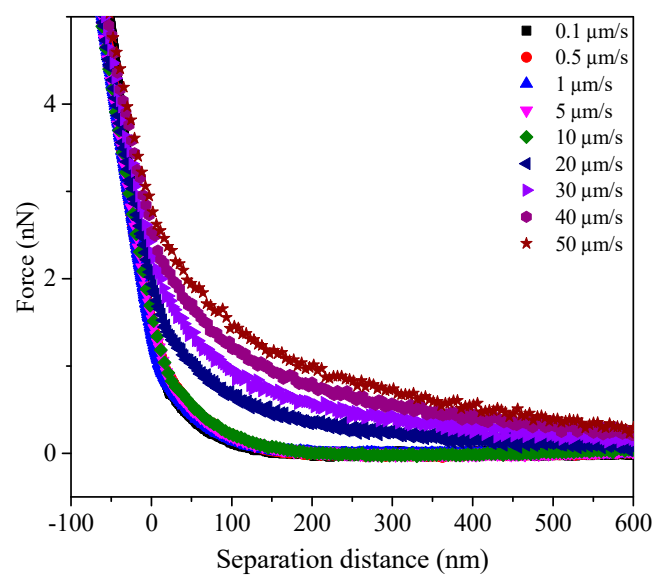

Figure 4. Interaction force between hydrophilic glass microsphere and bubble with different collision velocities.

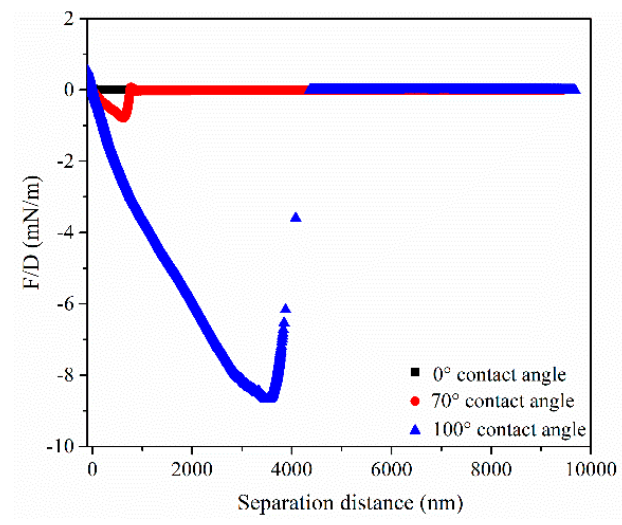

(a)

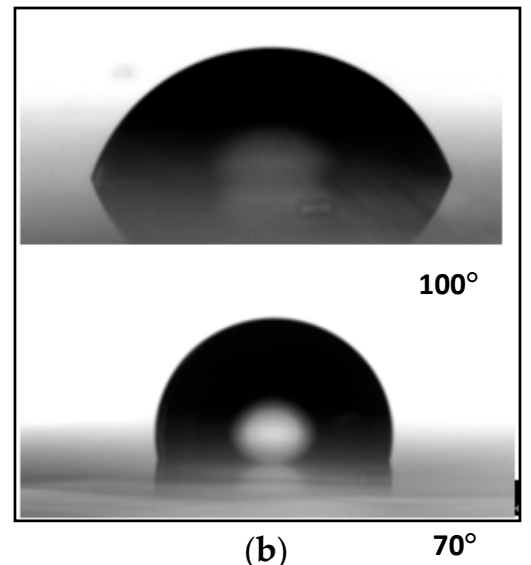

(b)

Figure 5. Effect of contact angle on the interaction force between glass microsphere and bubble with $1 \mu \mathrm{m} / \mathrm{s}$ approach velocity (a). The right insets are the contact angle image (b).

\subsection{Bubble-Particle Interaction Force in the Presence of DTAB and SDS}

The effect of DTAB and SDS on the interaction force between the hydrophilic glass microsphere and a bubble is shown in Figure 6. The approach velocity was fixed at $1 \mu \mathrm{m} / \mathrm{s}$. When $1 \mathrm{mM}$ of DTAB was added, a significant jump-into contact was observed in the AFM force curve, which indicated that a hydrophobic force was induced and overcame the repulsive DLVO force. When the DTAB concentration was $8 \mathrm{mM}$, the jump-into contact disappeared, and the repulsive force redominated the bubble-particle interaction. For SDS, there was no jump-into contact in the entire experimental concentration range. The repulsive force increased with the SDS concentration. The particle could not 
attach onto the bubble surface in the presence of SDS. The adsorption conformations of DTAB and SDS on the glass surface are shown in Figure 7. At low DTAB concentration, the cationic head group of DTAB adsorbed onto the negatively charged glass surface as a monolayer, and the hydrophobic group was oriented towards the water phase, making the glass surface hydrophobic. Therefore, an attractive hydrophobic force was induced, triggering film rupture. When the DTAB concentration was further increased, the DTAB molecules began to adsorb as a bilayer, reverting to a hydrophilic surface [16]. Meanwhile, the hydrophobicity of the air-water interface also decreased due to DTAB adsorption. The hydrophobic force disappeared and the water film between the bubble and particle was stabilised under the repulsive double-layer force between the head groups of DTAB. For the anionic SDS, the SDS molecules could not adsorb onto the negatively charged glass surface due to the repulsive double-layer force. However, the surface potentials of both the glass and air bubble decreased, resulting in an increase in the total repulsive force.

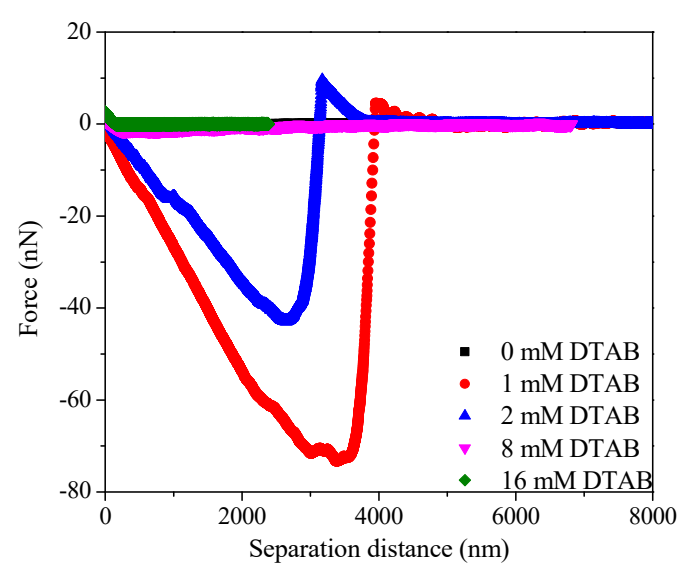

(a)

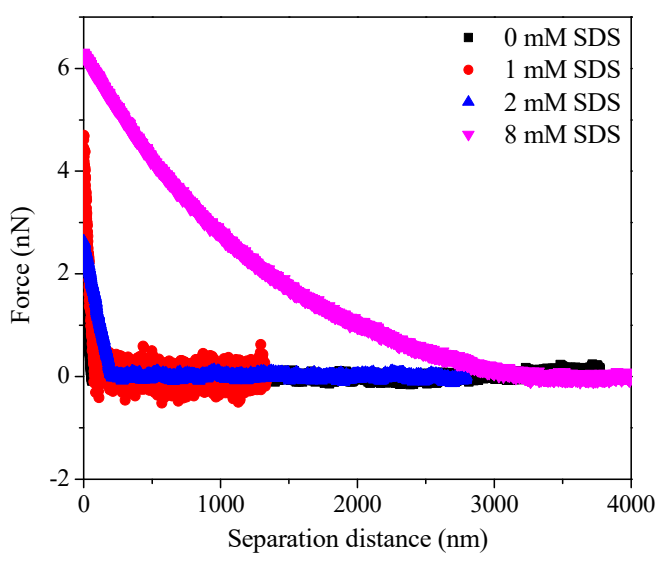

(b)

Figure 6. Effect of DTAB (a) and SDS (b) on the interaction force between hydrophilic glass microsphere and bubble.

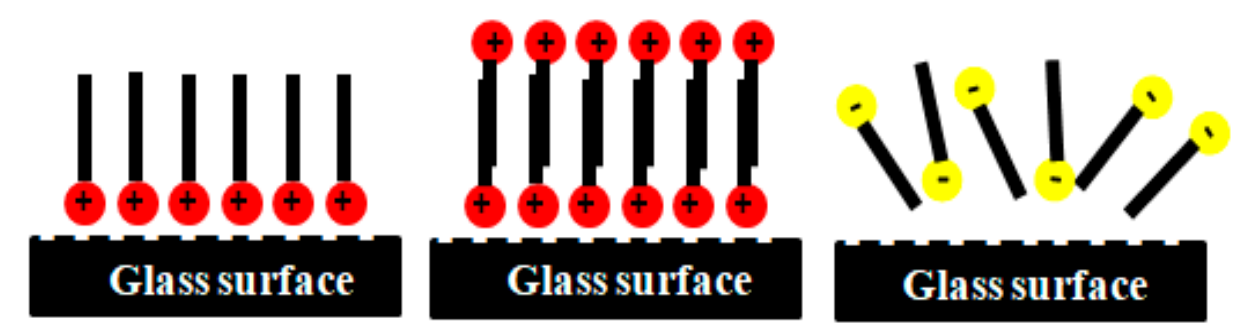

Figure 7. Adsorption conformations of DTAB and SDS on glass surface. The red ball represents the head group of DTAB and the yellow ball represents the head group of SDS.

The effect of DTAB and SDS on the interaction force between the hydrophobic glass microsphere ( $100^{\circ}$ contact angle) and a bubble is shown in Figure 8 . The approach velocity was fixed at $1 \mu \mathrm{m} / \mathrm{s}$. With increasing DTAB concentration, the jump-into distance decreased. At $8 \mathrm{mM}$ DTAB, the attractive hydrophobic force disappeared, and the repulsive force dominated the interaction. On one hand, the hydrophobicity of the hydrocarbon chain in DTAB was weaker than that in OTS. Therefore, the adsorption of DTAB reduced the water contact-angle of the hydrophobic glass microsphere. On the other hand, the decrease in surface tension contributed to the decrease in hydrophobicity. As a result, the hydrophobic force decreased on adding DTAB. At high DTAB concentration, the formation of the bilayer rendered the particle hydrophilic. In the case of SDS, only $1 \mathrm{mM}$ SDS was sufficient to screen the hydrophobic force. It should be noted that the increase in the repulsive double-layer force may also have led to the disappearance of the jump-into contact in the force curves. 


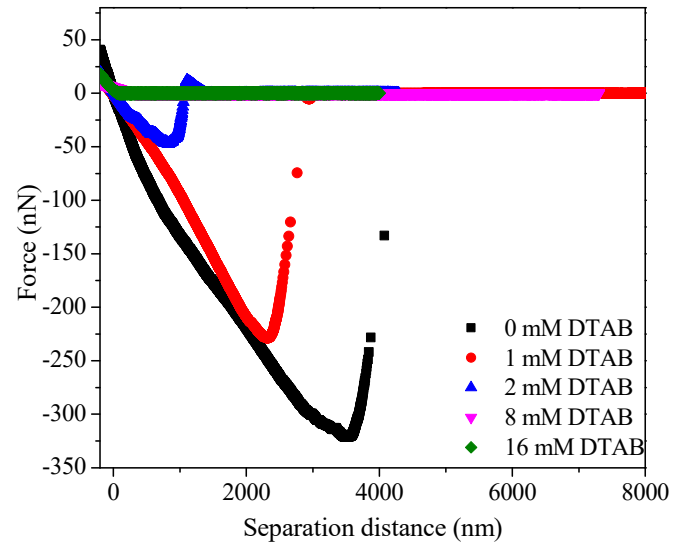

(a)

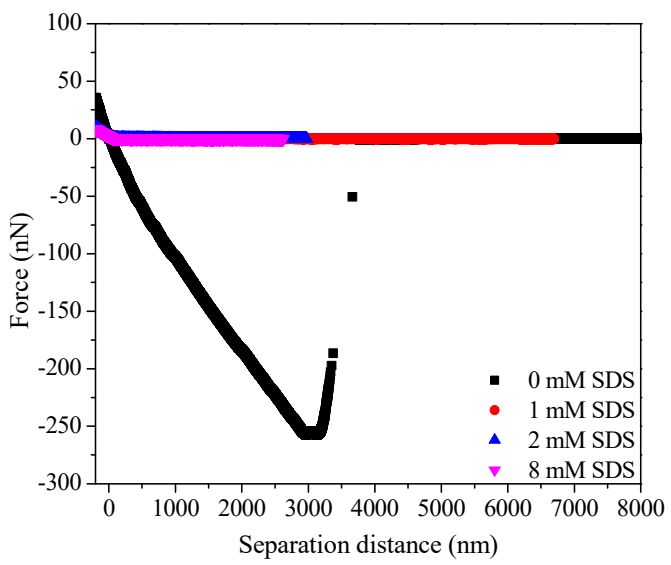

(b)

Figure 8. Effect of DTAB (a) and SDS (b) on the interaction force between hydrophobic glass microsphere $\left(100^{\circ}\right.$ contact angle) and bubble.

\subsection{Effect of DTAB and SDS on the Attachment Behaviour and Contact Angle}

The effect of DTAB and SDS on the attachment behaviour between the hydrophilic/hydrophobic glass and bubbles are shown in Figures 9 and 10. For hydrophilic glass, in the presence of 0, 8, and $16 \mathrm{mM}$ DTAB, when the contact time was set as $16 \mathrm{~s}$, no attachment was observed. In the presence of 1 and $2 \mathrm{mM}$ DTAB, the minimum contact time for successful bubble-particle attachment to be observed in our experimental system was $0.01 \mathrm{~s}$. This was consistent with the AFM results. The fast attachment at low DTAB concentrations was attributed to the induction of the hydrophobic force. In contrast, the bubble could not attach onto the glass surface with $16 \mathrm{~s}$ contact time at all the SDS concentrations. For hydrophobic glass, in the presence of 0,1 , and 2 mM DTAB, the minimum contact time for successful bubble-particle attachment to be observed was $0.01 \mathrm{~s}$. However, the length of the three-phase contact line decreased as DTAB concentration increased. This indicated that the attachment strength decreased. When the DTAB concentration was above $8 \mathrm{mM}$, there was no attachment even with $16 \mathrm{~s}$ contact time. In contrast, no attachment was observed at all the SDS concentrations. All the above findings were consistent with the AFM results.

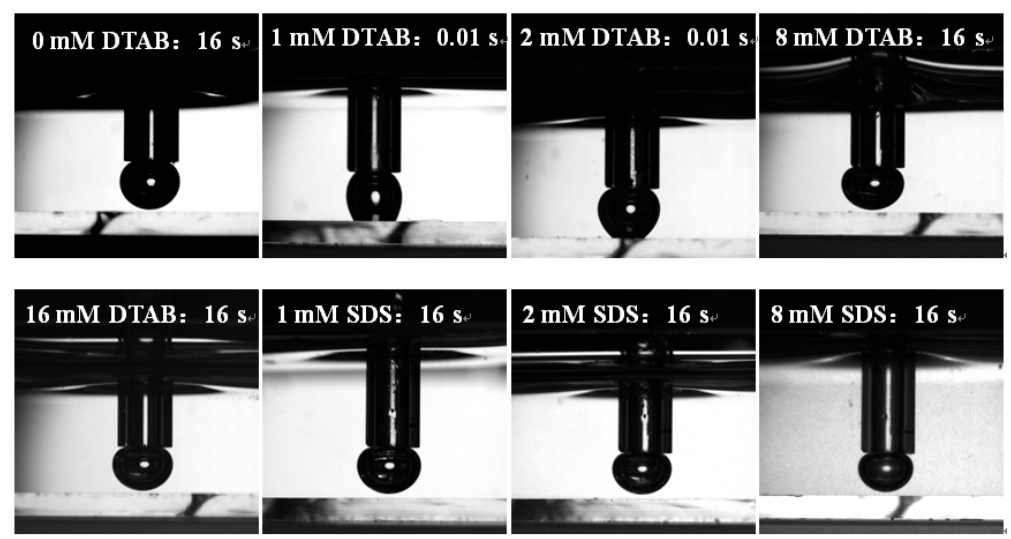

Figure 9. Effect of DTAB and SDS on the attachment behaviour between hydrophilic glass and bubble.

The effect of DTAB and SDS on the water contact-angle on the hydrophilic/hydrophobic glass surface is shown in Figure 11. For hydrophilic glass, with the increase in DTAB concentration, the water contact-angle initially increased and then decreased. The observed decrease in the contact angle was mainly due to the occurrence of double-layer adsorption. This variation trend in the contact angle was 
similar to that of the AFM results; however, some lag existed. For example, the maximum contact angle was observed at $8 \mathrm{mM}$ DTAB, while the hydrophobic force disappeared at this concentration. This is probably because DTAB also decreased the hydrophobicity of the air bubble. The hydrophobic force was the result of the combined interaction between the bubble and particle. In contrast, the water contact-angle decreased with the SDS concentration due to the decrease in surface tension, according to the Young's equation. For hydrophobic glass, the contact angle decreased with increasing DTAB/SDS concentration. The weaker hydrophobicity of the hydrocarbon chain in DTAB compared with that in OTS, and the decrease in surface tension, were the reasons for the decrease in the contact angle. In contrast, the decrease in the water contact-angle for SDS was due to the decrease in surface tension alone. It is notable that at low SDS concentrations ( 1 and $2 \mathrm{mM}$ ), the contact angle was still above $90^{\circ}$. It was speculated that the disappearance of the jump-into contact in the AFM force curves at low SDS concentrations was due to the increase in the negative surface potential and repulsive electrical double-layer force.

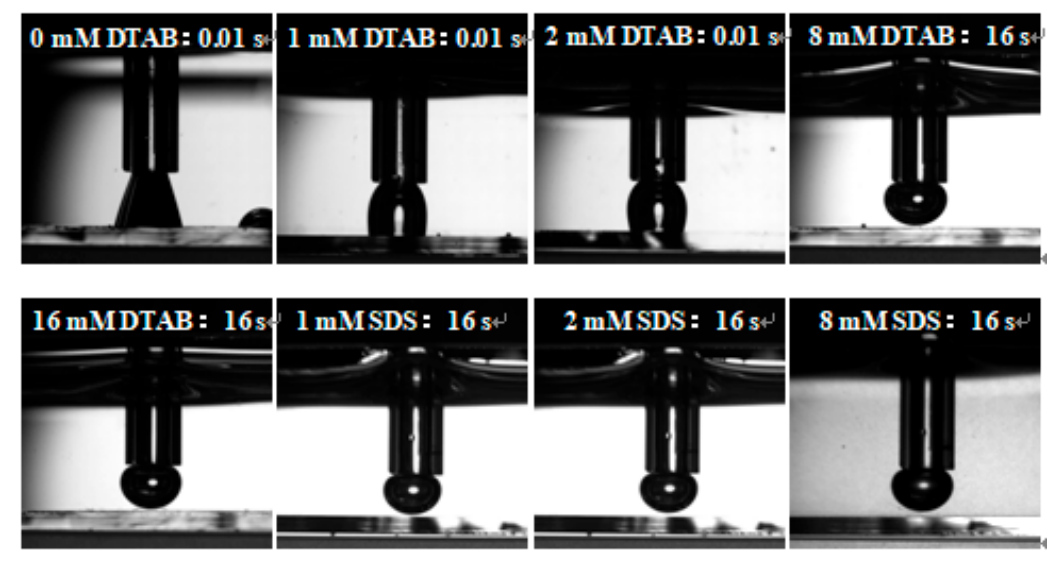

Figure 10. Effect of DTAB and SDS on the attachment behaviour between hydrophobic glass microsphere and bubble.

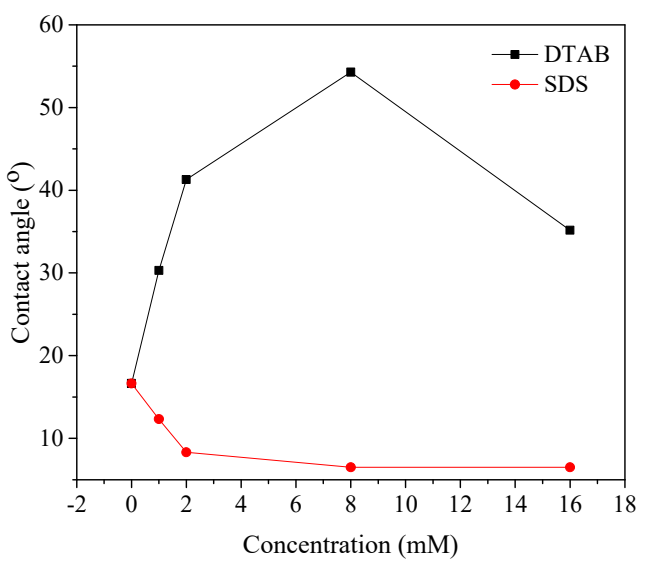

(a)

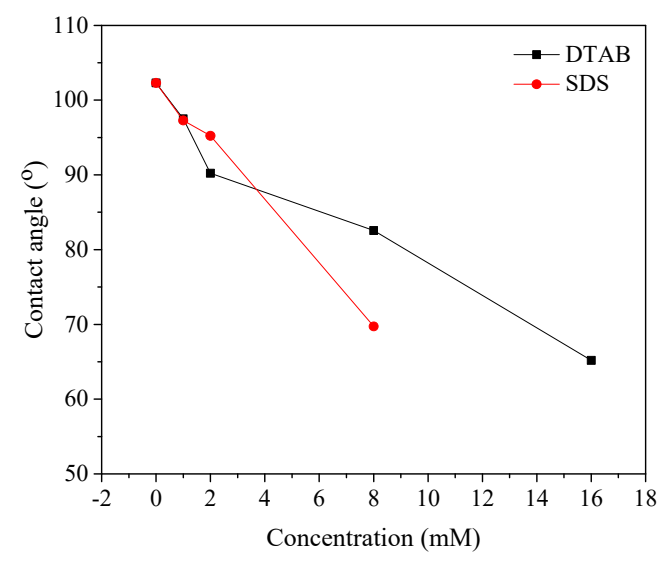

(b)

Figure 11. Effect of DTAB and SDS on the water contact angle of hydrophilic/hydrophobic glass surface. The left is hydrophilic glass (a) and the right is hydrophobic glass (b).

\section{Conclusions}

AFM and contact angle measurements were used to study the role of DTAB and SDS in bubble-particle attachment. The following conclusions were obtained: 
(1) The forces between the bubble and hydrophilic glass particle were always repulsive in the absence of DTAB and SDS. An attractive hydrophobic force was induced when the particle became hydrophobic, and the force was proportional to the water contact-angle.

(2) In the presence of DTAB and SDS, an attractive hydrophobic force could be induced between the hydrophilic particle and the bubble, triggering film rupture. However, at high DTAB concentration, the hydrophobic force disappeared, and the water film between the bubble and particle was stabilised under the repulsive double-layer force. The anionic SDS molecules could not adsorb onto the hydrophilic glass surface. The repulsive force always dominated the bubble-particle interaction.

(3) For hydrophobic glass, the hydrophobic force decreased, and even disappeared, with the addition of DTAB and SDS. All the findings from the AFM force curves were consistent with the attachment behaviour and contact angle results.

Author Contributions: Y.X. conceived and designed the experiments; Y.X., M.X., W.J., and M.L. performed the experiments; Y.X., X.G., and Y.C. analysed the data; Y.X. wrote the paper.

Funding: This work was supported by the Fundamental Research Funds for the Central Universities (2017CXNL04).

Conflicts of Interest: The authors declare no conflicts of interest.

\section{References}

1. Xing, Y.; Gui, X.; Pan, L.; Pinchasik, B.; Cao, Y.; Liu, J.; Kappl, M.; Butt, H. Recent experimental advances for understanding bubble-particle attachment in flotation. Adv. Colloid Interface Sci. 2017, 246, 105-142. [CrossRef] [PubMed]

2. Xing, Y.; Gui, X.; Cao, Y. The hydrophobic force for bubble-particle attachment in flotation-a brief review. Phys. Chem. Chem. Phys. 2017, 19, 24421-24435. [CrossRef] [PubMed]

3. Xing, Y.W.; Gui, X.H.; Cao, Y.J. Effect of calcium ion on coal flotation in the presence of kaolinite clay. Energy Fuels 2016, 30, 1517-1523. [CrossRef]

4. Xing, Y.W.; Gui, X.H.; Cao, Y.J.; Wang, D.P.; Zhang, H.J. Clean low-rank-coal purification technique combining cyclonic-static microbubble flotation column with collector emulsification. J. Clean. Prod. 2017, 153, 657-672. [CrossRef]

5. Xing, Y.; Xu, M.; Gui, X.; Cao, Y.; Babel, B.; Rudolph, M.; Weber, S.; Kappl, M.; Butt, H. The application of atomic force microscopy in mineral flotation. Adv. Colloid Interface Sci. 2018, 256, 373-392. [CrossRef] [PubMed]

6. Albijanic, B.; Ozdemir, O.; Nguyen, A.V.; Bradshaw, D. A review of induction and attachment times of wetting thin films between air bubbles and particles and its relevance in the separation of particles by flotation. Adv. Colloid Interface Sci. 2010, 159, 1-21. [CrossRef] [PubMed]

7. Ralston, J.; Fornasiero, D.; Mishchuk, N. The hydrophobic force in flotation-a critique. Colloids Surf. A Physicochem. Eng. Asp. 2001, 192, 39-51. [CrossRef]

8. Meyer, E.E.; Rosenberg, K.J.; Israelachvili, J. Recent progress in understanding hydrophobic interactions. Proc. Natl. Acad. Sci. USA 2006, 103, 15739-15746. [CrossRef] [PubMed]

9. Pan, L.; Jung, S.; Yoon, R.H. Effect of hydrophobicity on the stability of the wetting films of water formed on gold surfaces. J. Colloid Interface Sci. 2011, 361, 321-330. [CrossRef] [PubMed]

10. Xing, Y.; Gui, X.; Karakas, F.; Cao, Y. Role of Collectors and Depressants in Mineral Flotation: A Theoretical Analysis Based on Extended DLVO Theory. Minerals 2017, 7, 223. [CrossRef]

11. Xing, Y.; Gui, X.; Cao, Y. Effect of bubble size on bubble-particle attachment and film drainage kinetics-A theoretical study. Powder Technol. 2017, 322, 140-146. [CrossRef]

12. Pan, L.; Yoon, R. Measurement of hydrophobic forces in thin liquid films of water between bubbles and xanthate-treated gold surfaces. Miner. Eng. 2016, 98, 240-250. [CrossRef]

13. Butt, H. A technique for measuring the force between a colloidal particle in water and a bubble. J. Colloid Interface Sci. 1994, 166, 109-117. [CrossRef]

14. Nguyen, A.; Nalaskowski, J.; Miller, J. A study of bubble-particle interaction using atomic force microscopy. Miner. Eng. 2003, 16, 1173-1181. [CrossRef] 
15. Nguyen, A.; Evans, G.; Nalaskowski, J.; Miller, J. Hydrodynamic interaction between an air bubble and a particle: Atomic force microscopy measurements. Exp. Therm. Fluid Sci. 2004, 28, 387-394. [CrossRef]

16. Preuss, M.; Butt, H.-J. Direct measurement of particle-bubble interactions in aqueous electrolyte: Dependence on surfactant. Langmuir 1998, 14, 3164-3174. [CrossRef]

17. Xu, M.D.; Xing, Y.W.; Cao, Y.J.; Gui, X.H. Effect of dodecane and oleic acid on the attachment between oxidized coal and bubbles. Minerals 2018, 8, 29. [CrossRef]

18. Tabor, R.F.; Grieser, F.; Dagastine, R.R.; Chan, D.Y. Measurement and analysis of forces in bubble and droplet systems using AFM. J. Colloid Interface Sci. 2012, 371, 1-14. [CrossRef] [PubMed]

19. Johnson, D.J.; Miles, N.J.; Hilal, N. Quantification of particle-bubble interactions using atomic force microscopy: A review. Adv. Colloid Interface Sci. 2006, 127, 67-81. [CrossRef] [PubMed]

20. Assemi, S.; Nguyen, A.V.; Miller, J.D. Direct measurement of particle-bubble interaction forces using atomic force microscopy. Int. J. Miner. Proc. 2008, 89, 65-70. [CrossRef]

21. Preuss, M.; Butt, H.J. Direct measurement of forces between particles and bubbles. Int. J. Miner. Proc. 1999, 56, 99-115. [CrossRef]

(C) 2018 by the authors. Licensee MDPI, Basel, Switzerland. This article is an open access article distributed under the terms and conditions of the Creative Commons Attribution (CC BY) license (http:/ / creativecommons.org/licenses/by/4.0/). 